Internat. J. Math. \& Math. Sci.

Vol. 22, No. 2 (1999) 271-279

S 0161-1712<99>22271-5

(c) Electronic Publishing House

\title{
EXISTENCE AND UNIQUENESS THEOREM FOR A SOLUTION OF FUZZY DIFFERENTIAL EQUATIONS
}

\author{
JONG YEOUL PARK and HYO KEUN HAN
}

(Received 10 June 1996)

\begin{abstract}
By using the method of successive approximation, we prove the existence and uniqueness of a solution of the fuzzy differential equation $x^{\prime}(t)=f(t, x(t)), x\left(t_{0}\right)=x_{0}$. We also consider an $\epsilon$-approximate solution of the above fuzzy differential equation.
\end{abstract}

Keywords and phrases. Fuzzy set-valued mapping, levelwise continuous, fuzzy derivative, fuzzy integral, fuzzy differential equation, fuzzy solution, fuzzy $\epsilon$-approximate solution.

1991 Mathematics Subject Classification. 04A72, 34A45, 34A46.

1. Introduction. The differential equation

$$
x^{\prime}(t)=f(t, x(t)), \quad x\left(t_{0}\right)=x_{0}
$$

has a solution provided $f$ is continuous and satisfies a Lipschitz condition by $\mathrm{C}$. Corduneanu [2]. The definition given here generalizes that of Aumann [1] for setvalued mappings. Kaleva [3] discussed the properties of differentiable fuzzy set-valued mappings and gave the existence and uniqueness theorem for a solution of the fuzzy differential equation $x^{\prime}(t)=f(t, x(t))$ when $f$ satisfies the Lipschitz condition. Also, in [4], he dealt with fuzzy differential equations on locally compact spaces. Park [6, 7] showed existence of solutions for fuzzy integral equations and a fixed point theorem for a pair of generalized nonexpansive fuzzy mappings.

In this paper, we prove the existence and uniqueness theorem of a solution to the fuzzy differential equation (1.1), where $f: I \times E^{n} \rightarrow E^{n}$ is levelwise continuous and satisfies a generalized Lipschitz condition.

Under some hypotheses, we consider an $\epsilon$-approximate solution of the above fuzzy differential equation.

2. Preliminaries. Let $P_{K}\left(R^{n}\right)$ denote the family of all nonempty compact convex subsets of $R^{n}$ and define the addition and scalar multiplication in $P_{K}\left(R^{n}\right)$ as usual. Let $A$ and $B$ be two nonempty bounded subsets of $R^{n}$. The distance between $A$ and $B$ is defined by the Hausdorff metric

$$
d(A, B)=\max \left\{\sup _{a \in A} \inf _{b \in B}\|a-b\|, \sup _{b \in B} \inf _{a \in A}\|a-b\|\right\},
$$

where $\|\cdot\|$ denotes the usual Euclidean norm in $R^{n}$. Then it is clear that $\left(P_{K}\left(R^{n}\right), d\right)$ becomes a metric space.

THEOREM 2.1 [8]. The metric space $\left(P_{K}\left(R^{n}\right), d\right)$ is complete and separable. 
Let $T=[c, d] \subset R$ be a compact interval and denote

$$
E^{n}=\left\{u: R^{n} \rightarrow[0,1] \mid u \text { satisfies (i)-(iv) below }\right\},
$$

where

(i) $u$ is normal, i.e., there exists an $x_{0} \in R^{n}$ such that $u\left(x_{0}\right)=1$,

(ii) $u$ is fuzzy convex,

(iii) $u$ is upper semicontinuous,

(iv) $[u]^{0}=\operatorname{cl}\left\{x \in R^{n} \mid u(x)>0\right\}$ is compact.

For $0<\alpha \leq 1$, denote $[u]^{\alpha}=\left\{x \in R^{n} \mid u(x) \geq \alpha\right\}$, then from (i)-(iv), it follows that the $\alpha$-level set $[u]^{\alpha} \in P_{K}\left(R^{n}\right)$ for all $0 \leq \alpha \leq 1$.

If $g: R^{n} \times R^{n} \rightarrow R^{n}$ is a function, then, according to Zadeh's extension principle, we can extend $g$ to $E^{n} \times E^{n} \rightarrow E^{n}$ by the equation

$$
g(u, v)(z)=\sup _{z=g(x, y)} \min \{u(x), v(y)\} .
$$

It is well known that

$$
[g(u, v)]^{\alpha}=g\left([u]^{\alpha},[v]^{\alpha}\right)
$$

for all $u, v \in E^{n}, 0 \leq \alpha \leq 1$ and $g$ is continuous. Especially for addition and scalar multiplication, we have

$$
[u+v]^{\alpha}=[u]^{\alpha}+[v]^{\alpha}, \quad[k u]^{\alpha}=k[u]^{\alpha},
$$

where $u, v \in E^{n}, k \in R, 0 \leq \alpha \leq 1$.

THEOREM 2.2 [5]. If $u \in E^{n}$, then

(1) $[u]^{\alpha} \in P_{K}\left(R^{n}\right)$ for all $0 \leq \alpha \leq 1$,

(2) $[u]^{\alpha} \subset[u]^{\alpha_{1}}$ for all $0 \leq \alpha_{1} \leq \alpha_{2} \leq 1$,

(3) if $\left\{\alpha_{k}\right\} \subset[0,1]$ is a nondecreasing sequence converging to $\alpha>0$, then

$$
[u]^{\alpha}=\bigcap_{k \geq 1}[u]^{\alpha_{k}}
$$

Conversely, if $\left\{A^{\alpha} \mid 0 \leq \alpha \leq 1\right\}$ is a family of subsets of $R^{n}$ satisfying (1)-(3), then there exists $u \in E^{n}$ such that

$$
[u]^{\alpha}=A^{\alpha} \quad \text { for } 0<\alpha \leq 1
$$

and

$$
[u]^{0}=\overline{\bigcup_{0<\alpha \leq 1} A^{\alpha}} \subset A^{0} .
$$

Define $D: E^{n} \times E^{n} \rightarrow R^{+} \cup\{0\}$ by the equation

$$
D(u, v)=\sup _{0 \leq \alpha \leq 1} d\left([u]^{\alpha},[v]^{\alpha}\right),
$$

where $d$ is the Hausdorff metric defined in $P_{K}\left(R^{n}\right)$.

The following definitions and theorems are given in [3].

DEFinition 2.1. A mapping $F: T \rightarrow E^{n}$ is strongly measurable if, for all $\alpha \in[0,1]$, the set-valued mapping $F_{\alpha}: T \rightarrow P_{K}\left(R^{n}\right)$ defined by

$$
F_{\alpha}(t)=[F(t)]^{\alpha}
$$


is Lebesgue measurable, when $P_{K}\left(R^{n}\right)$ is endowed with the topology generated by the Hausdorff metric $d$.

DefinITION 2.2. A mapping $F: T \rightarrow E^{n}$ is called levelwise continuous at $t_{0} \in T$ if the set-valued mapping $F_{\alpha}(t)=[F(t)]^{\alpha}$ is continuous at $t=t_{0}$ with respect to the Hausdorff metric $d$ for all $\alpha \in[0,1]$.

A mapping $F: T \rightarrow E^{n}$ is called integrably bounded if there exists an integrable function $h$ such that $\|x\| \leq h(t)$ for all $x \in F_{0}(t)$.

Definition 2.3. Let $F: T \rightarrow E^{n}$. The integral of $F$ over $T$, denoted by $\int_{T} F(t)$ or $\int_{c}^{d} F(t) d t$, is defined levelwise by the equation

$$
\begin{aligned}
\left(\int_{T} F(t) d t\right)^{\alpha} & =\int_{T} F_{\alpha}(t) d t \\
& =\left\{\int_{T} f(t) d t \mid f: T \rightarrow R^{n} \text { is a measurable selection for } F_{\alpha}\right\}
\end{aligned}
$$

for all $0<\alpha \leq 1$.

A strongly measurable and integrably bounded mapping $F: T \rightarrow E^{n}$ is said to be integrable over $T$ if $\int_{T} F(t) d t \in E^{n}$.

THEOREM 2.3. If $F: T \rightarrow E^{n}$ is strongly measurable and integrably bounded, then $F$ is integrable.

It is known that $\left[\int_{T} F(t) d t\right]^{0}=\int_{T} F_{0}(t) d t$.

THEOREM 2.4. Let $F, G: T \rightarrow E^{n}$ be integrable, and $\lambda \in R$. Then

(i) $\int_{T}(F(t)+G(t)) d t=\int_{T} F(t) d t+\int_{T} G(t) d t$.

(ii) $\int_{T} \lambda F(t) d t=\lambda \int_{T} F(t) d t$.

(iii) $D(F, G)$ is integrable.

(iv) $D\left(\int_{T} F(t) d t, \int_{T} G(t) d t\right) \leq \int_{T} D(F, G)(t) d t$.

Definition 2.4. A mapping $F: T \rightarrow E^{n}$ is called differentiable at $t_{0} \in T$ if, for any $\alpha \in[0,1]$, the set-valued mapping $F_{\alpha}(t)=[F(t)]^{\alpha}$ is Hukuhara differentiable at point $t_{0}$ with $D F_{\alpha}\left(t_{0}\right)$ and the family $\left\{D F_{\alpha}\left(t_{0}\right) \mid \alpha \in[0,1]\right\}$ define a fuzzy number $F\left(t_{0}\right) \in E^{n}$.

If $F: T \rightarrow E^{n}$ is differentiable at $t_{0} \in T$, then we say that $F^{\prime}\left(t_{0}\right)$ is the fuzzy derivative of $F(t)$ at the point $t_{0}$.

THEOREM 2.5. Let $F: T \rightarrow E^{1}$ be differentiable. Denote $F_{\alpha}(t)=\left[f_{\alpha}(t), g_{\alpha}(t)\right]$. Then $f_{\alpha}$ and $g_{\alpha}$ are differentiable and $\left[F^{\prime}(t)\right]^{\alpha}=\left[f_{\alpha}^{\prime}(t), g_{\alpha}^{\prime}(t)\right]$.

THEOREM 2.6. Let $F: T \rightarrow E^{n}$ be differentiable and assume that the derivative $F^{\prime}$ is integrable over $T$. Then, for each $s \in T$, we have

$$
F(s)=F(a)+\int_{a}^{s} F^{\prime}(t) d t .
$$

DEFINITION 2.5. A mapping $f: T \times E^{n} \rightarrow E^{n}$ is called levelwise continuous at point $\left(t_{0}, x_{0}\right) \in T \times E^{n}$ provided, for any fixed $\alpha \in[0,1]$ and arbitrary $\epsilon>0$, there exists a 
$\delta(\epsilon, \alpha)>0$ such that

$$
d\left([f(t, x)]^{\alpha},\left[f\left(t_{0}, x_{0}\right)\right]^{\alpha}\right)<\epsilon
$$

whenever $\left|t-t_{0}\right|<\delta(\epsilon, \alpha)$ and $d\left([x]^{\alpha},\left[x_{0}\right]^{\alpha}\right)<\delta(\epsilon, \alpha)$ for all $t \in T, x \in E^{n}$.

3. Fuzzy differential equations. Assume that $f: I \times E^{n} \rightarrow E^{n}$ is levelwise continuous, where the interval $I=\left\{t:\left|t-t_{0}\right| \leq \delta \leq a\right\}$. Consider the fuzzy differential equation (1.1) where $x_{0} \in E^{n}$. We denote $J_{0}=I \times B\left(x_{0}, b\right)$, where $a>0, b>0, x_{0} \in E^{n}$,

$$
B\left(x_{0}, b\right)=\left\{x \in E^{n} \mid D\left(x, x_{0}\right) \leq b\right\} .
$$

DEFINITION 3.1. A mapping $x: I \rightarrow E^{n}$ is a solution to the problem (1.1) if it is levelwise continuous and satisfies the integral equation

$$
x(t)=x_{0}+\int_{t_{0}}^{t} f(s, x(s)) d s \text { for all } t \in I .
$$

According to the method of successive approximation, let us consider the sequence $\left\{x_{n}(t)\right\}$ such that

$$
x_{n}(t)=x_{0}+\int_{t_{0}}^{t} f\left(s, x_{n-1}(s)\right) d s, \quad n=1,2, \ldots,
$$

where $x_{0}(t) \equiv x_{0}, t \in I$.

THEOREM 3.1. Assume that

(i) a mapping $f: J_{0} \rightarrow E^{n}$ is levelwise continuous,

(ii) for any pair $(t, x),(t, y) \in J_{0}$, we have

$$
d\left([f(t, x)]^{\alpha},[f(t, y)]^{\alpha}\right) \leq \operatorname{Ld}\left([x]^{\alpha},[y]^{\alpha}\right),
$$

where $L>0$ is a given constant and for any $\alpha \in[0,1]$.

Then there exists a unique solution $x=x(t)$ of (1.1) defined on the interval

$$
\left|t-t_{0}\right| \leq \delta=\min \left\{a, \frac{b}{M}\right\}
$$

where $M=D(f(t, x), \hat{o}), \hat{o} \in E^{n}$ such that $\hat{o}(t)=1$ for $t=0$ and 0 otherwise and for any $(t, x) \in J_{0}$.

Moreover, there exists a fuzzy set-valued mapping $x: I \rightarrow E^{n}$ such that $D\left(x_{n}(t), x(t)\right)$ $\rightarrow 0$ on $\left|t-t_{0}\right| \leq \delta$ as $n \rightarrow \infty$.

Proof. Let $t \in I$, from (3.3), it follows that, for $n=1$,

$$
x_{1}(t)=x_{0}+\int_{t_{0}}^{t} f\left(s, x_{0}\right) d s
$$

which proves that $x(t)$ is levelwise continuous on $\left|t-t_{0}\right| \leq a$ and, hence on $\left|t-t_{0}\right| \leq \delta$. Moreover, for any $\alpha \in[0,1]$, we have

$$
d\left(\left[x_{1}(t)\right]^{\alpha},\left[x_{0}\right]^{\alpha}\right)=d\left(\left[\int_{t_{0}}^{t} f\left(s, x_{0}\right) d s\right]^{\alpha}, 0\right) \leq \int_{t_{0}}^{t} d\left(\left[f\left(s, x_{0}\right)\right]^{\alpha}, 0\right) d s
$$

and by the definition of $D$, we get

$$
D\left(x_{1}(t), x_{0}\right) \leq M\left|t-t_{0}\right| \leq M \delta=b
$$


if $\left|t-t_{0}\right| \leq \delta$, where $M=D(f(t, x), \hat{o}), \hat{o} \in E^{n}$ and for any $(t, x) \in J_{0}$.

Now, assume that $x_{n-1}(t)$ is levelwise continuous on $\left|t-t_{0}\right| \leq \delta$ and that

$$
D\left(x_{n-1}(t), x_{0}\right) \leq M\left|t-t_{0}\right| \leq M \delta=b
$$

if $\left|t-t_{0}\right| \leq \delta$, where $M=D(f(t, x), \hat{o}), \hat{o} \in E^{n}$ and for any $(t, x) \in J_{0}$.

From (3.3), we deduce that $x_{n}(t)$ is levelwise continuous on $\left|t-t_{0}\right| \leq \delta$ and that

$$
D\left(x_{n}(t), x_{0}\right) \leq M\left|t-t_{0}\right| \leq M \delta=b
$$

if $\left|t-t_{0}\right| \leq \delta$, where $M=D(f(t, x), \hat{o}), \hat{o} \in E^{n}$ and for any $(t, y) \in J_{0}$.

Consequently, we conclude that $\left\{x_{n}(t)\right\}$ consists of levelwise continuous mappings on $\left|t-t_{0}\right| \leq \delta$ and that

$$
\left(t, x_{n}(t)\right) \in J_{0}, \quad\left|t-t_{0}\right| \leq \delta, \quad n=1,2, \ldots
$$

Let us prove that there exists a fuzzy set-valued mapping $x: I \rightarrow E^{n}$ such that $D\left(x_{n}(t)\right.$, $x(t)) \rightarrow 0$ uniformly on $\left|t-t_{0}\right| \leq \delta$ as $n \rightarrow \infty$. For $n=2$, from (3.3),

$$
x_{2}(t)=x_{0}+\int_{t_{0}}^{t} f\left(s, x_{1}(s)\right) d s .
$$

From (3.6) and (3.12), we have

$$
\begin{aligned}
d\left(\left[x_{2}(t)\right]^{\alpha},\left[x_{1}(t)\right]^{\alpha}\right) & =d\left(\left[\int_{t_{0}}^{t} f\left(s, x_{1}(s)\right) d s\right]^{\alpha},\left[\int_{t_{0}}^{t} f\left(s, x_{0}\right) d s\right]^{\alpha}\right) \\
& \leq \int_{t_{0}}^{t} d\left(\left[f\left(s, x_{1}(s)\right)\right]^{\alpha},\left[f\left(s, x_{0}\right)\right]^{\alpha}\right) d s
\end{aligned}
$$

for any $\alpha \in[0,1]$.

According to the condition (3.4), we obtain

$$
d\left(\left[x_{2}(t)\right]^{\alpha},\left[x_{1}(t)\right]^{\alpha}\right) \leq \int_{t_{0}}^{t} \operatorname{Ld}\left(\left[x_{1}(s)\right]^{\alpha},\left[x_{0}\right]^{\alpha}\right) d s
$$

and by the definition of $D$, we obtain

$$
D\left(x_{2}(t), x_{1}(t)\right) \leq L \int_{t_{0}}^{t} D\left(x_{1}(s), x_{0}(s)\right) d s .
$$

Now, we can apply the first inequality (3.8) in the right-hand side of (3.15) to get

$$
D\left(x_{2}(t), x_{1}(t)\right) \leq M L \frac{\left|t-t_{0}\right|^{2}}{2 !} \leq M L \frac{\delta^{2}}{2 !} .
$$

Starting from (3.8) and (3.16), assume that

$$
D\left(x_{n}(t), x_{n-1}(t)\right) \leq M L^{n-1} \frac{\left|t-t_{0}\right|^{n}}{n !} \leq M L^{n-1} \frac{\delta^{n}}{n !}
$$

and let us prove that such an inequality holds for $D\left(x_{n+1}(t), x_{n}(t)\right)$. 
Indeed, from (3.3) and condition (3.4), it follows that

$$
\begin{aligned}
d\left(\left[x_{n+1}(t)\right]^{\alpha},\left[x_{n}(t)\right]^{\alpha}\right) & =d\left(\left[\int_{t_{0}}^{t} f\left(s, x_{n}(s)\right) d s\right]^{\alpha},\left[\int_{t_{0}}^{t} f\left(s, x_{n-1}(s)\right) d s\right]^{\alpha}\right) \\
& \leq \int_{t_{0}}^{t} d\left(\left[f\left(s, x_{n}(s)\right)\right]^{\alpha},\left[f\left(s, x_{n-1}(s)\right)\right]^{\alpha}\right) d s \\
& \leq \int_{t_{0}}^{t} L d\left(\left[x_{n}(s)\right]^{\alpha},\left[x_{n-1}(s)\right]^{\alpha}\right) d s
\end{aligned}
$$

for any $\alpha \in[0,1]$ and from the definition of $D$, we have

$$
D\left(x_{n+1}(t), x_{n}(t)\right) \leq L \int_{t_{0}}^{t} D\left(x_{n}(s), x_{n-1}(s)\right) d s .
$$

According to (3.17), we get

$$
D\left(x_{n+1}(t), x_{n}(t)\right) \leq M L^{n} \int_{t_{0}}^{t} \frac{\left|s-t_{0}\right|^{n}}{n !} d s=M L^{n} \frac{\left|t-t_{0}\right|^{n+1}}{(n+1) !} \leq M L^{n} \frac{\delta^{n+1}}{(n+1) !} .
$$

Consequently, inequality (3.17) holds for $n=1,2, \ldots$. We can also write

$$
D\left(x_{n}(t), x_{n-1}(t)\right) \leq \frac{M}{L} \frac{(L \delta)^{n}}{n !}
$$

for $n=1,2, \ldots$, and $\left|t-t_{0}\right| \leq \delta$.

Let us mention now that

$$
x_{n}(t)=x_{0}+\left[x_{1}(t)-x_{0}\right]+\cdots+\left[x_{n}(t)-x_{n-1}(t)\right],
$$

which implies that the sequence $\left\{x_{n}(t)\right\}$ and the series

$$
x_{0}+\sum_{n=1}^{\infty}\left[x_{n}(t)-x_{n-1}(t)\right]
$$

have the same convergence properties.

From (3.21), according to the convergence criterion of Weierstrass, it follows that the series having the general term $x_{n}(t)-x_{n-1}(t)$, so $D\left(x_{n}(t), x_{n-1}(t)\right) \rightarrow 0$ uniformly on $\left|t-t_{0}\right| \leq \delta$ as $n \rightarrow \infty$.

Hence, there exists a fuzzy set-valued mapping $x: I \rightarrow E^{n}$ such that $D\left(x_{n}(t), x(t)\right) \rightarrow$ 0 uniformly on $\left|t-t_{0}\right| \leq \delta$ as $n \rightarrow \infty$.

From (3.4), we get

$$
d\left(\left[f\left(t, x_{n}(t)\right)\right]^{\alpha},[f(t, x(t))]^{\alpha}\right) \leq L d\left(\left[x_{n}(t)\right]^{\alpha},[x(t)]^{\alpha}\right)
$$

for any $\alpha \in[0,1]$. By the definition of $D$,

$$
D\left(f\left(t, x_{n}(t)\right), f(t, x(t))\right) \leq L D\left(x_{n}(t), x(t)\right) \longrightarrow 0
$$

uniformly on $\left|t-t_{0}\right| \leq \delta$ as $n \rightarrow \infty$.

Taking (3.25) into account, from (3.3), we obtain, for $n \rightarrow \infty$,

$$
x(t)=x_{0}+\int_{t_{0}}^{t} f(s, x(s)) d s
$$


Consequently, there is at least one levelwise continuous solution of (1.1).

We want to prove now that this solution is unique, that is, from

$$
y(t)=x_{0}+\int_{t_{0}}^{t} f(s, y(s)) d s
$$

on $\left|t-t_{0}\right| \leq \delta$, it follows that $D(x(t), y(t)) \equiv 0$. Indeed, from (3.3) and (3.27), we obtain

$$
\begin{aligned}
d\left([y(t)]^{\alpha},\left[x_{n}(t)\right]^{\alpha}\right) & =d\left(\left[\int_{t_{0}}^{t} f(s, y(s)) d s\right]^{\alpha},\left[\int_{t_{0}}^{t} f\left(s, x_{n-1}(s)\right) d s\right]^{\alpha}\right) \\
& \leq \int_{t_{0}}^{t} d\left([f(s, y(s))]^{\alpha},\left[f\left(s, x_{n-1}(s)\right)\right]^{\alpha}\right) d s \\
& \leq \int_{t_{0}}^{t} L d\left([y(s)]^{\alpha},\left[x_{n-1}(s)\right]^{\alpha}\right) d s
\end{aligned}
$$

for any $\alpha \in[0,1], n=1,2, \ldots$.

By the definition of $D$, we obtain

$$
D\left(y(t), x_{n}(t)\right) \leq L \int_{t_{0}}^{t} D\left(y(s), x_{n-1}(s)\right) d s, \quad n=1,2, \ldots .
$$

But $D\left(y(t), x_{0}\right) \leq b$ on $\left|t-t_{0}\right| \leq \delta, y(t)$ being a solution of (3.27). It follows from (3.29) that

$$
D\left(y(t), x_{1}(t)\right) \leq b L\left|t-t_{0}\right|
$$

on $\left|t-t_{0}\right| \leq \delta$. Now, assume that

$$
D\left(y(t), x_{n}(t)\right) \leq b L^{n} \frac{\left|t-t_{0}\right|^{n}}{n !}
$$

on the interval $\left|t-t_{0}\right| \leq \delta$. From

$$
D\left(y(t), x_{n+1}(t)\right) \leq L \int_{t_{0}}^{t} D\left(y(s), x_{n}(s)\right) d s
$$

and (3.31), one obtains

$$
D\left(y(t), x_{n+1}(t)\right) \leq b L^{n+1} \frac{\left|t-t_{0}\right|^{n+1}}{(n+1) !} .
$$

Consequently, (3.31) holds for any $n$, which leads to the conclusion

$$
D\left(y(t), x_{n}(t)\right)=D\left(x(t), x_{n}(t)\right) \longrightarrow 0
$$

on the interval $\left|t-t_{0}\right| \leq \delta$ as $n \rightarrow \infty$.

This proves the uniqueness of the solution for (1.1).

DEFINITION 3.2. A mapping $x: L \rightarrow E^{n}$ is an $\epsilon$-approximate solution of (1.1) if the following properties hold

(a) $x(t)$ is levelwise continuous on $\left|t-t_{0}\right| \leq \delta$,

(b) the derivative $x^{\prime}(t)$ exists and it is levelwise continuous,

(c) for all $t$ for which $x^{\prime}(t)$ is defined, we have

$$
D\left(x^{\prime}(t), f(t, x(t))\right)<\epsilon .
$$


THEOREM 3.2. A mapping $f: J_{0} \rightarrow E^{n}$ is levelwise continuous, and let $\epsilon>0$ be arbitrary. Then there exists at least one $\epsilon$-approximate solution of (1.1), defined on $\left|t-t_{0}\right| \leq \delta=\min \{a, b / M\}$, where $M=D(f(t, x), \hat{o}), \hat{o} \in E^{n}$ and for any $(t, x) \in J_{0}$.

Proof. In as much as a mapping $f: J_{0} \rightarrow E^{n}$ is a levelwise continuous on a compact set $J_{0}$, it follows that $f(t, x)$ is uniformly levelwise continuous.

Consequently, for any $\alpha \in[0,1]$, we can find $\delta>0$ such that $d\left([f(t, x)]^{\alpha},[f(s, y)]^{\alpha}\right)$ $<\epsilon$.

Now, we construct the approximate solution for $t \in\left[t_{0}, t_{0}+\delta\right]$, the construction being completely similar for $t \in\left[t_{0}-\delta, t_{0}\right]$.

Let us consider a division

$$
t_{0}<t_{1}<\cdots<t_{n}=t_{0}+\delta
$$

of $\left[t_{0}, t_{0}+\delta\right]$ such that

$$
\max _{k}\left(t_{k}-t_{k-1}\right)<\lambda=\min \left\{\delta, \frac{\delta}{M}\right\} .
$$

We define a mapping $x: I \rightarrow E^{n}$ as follows

$$
\begin{gathered}
x\left(t_{0}\right)=x_{0}, \\
x(t)=x\left(t_{k}\right)+f\left(t_{k}, x\left(t_{k}\right)\right)\left(t-t_{k}\right)
\end{gathered}
$$

on $t_{k}<t \leq t_{k+1}, k=0,1, \ldots, n-1$.

It is obvious that a mapping $x: I \rightarrow E^{n}$ satisfies the first two properties from the definition of an $\epsilon$-approximate solution.

Now, we want to prove that the last property is also fulfilled. Indeed, $x^{\prime}(t)=f\left(t_{k}\right.$, $\left.x\left(t_{k}\right)\right)$ on $\left(t_{k}, t_{k+1}\right)$ and for any $\alpha \in[0,1]$,

$$
d\left(\left[x^{\prime}(t)\right]^{\alpha},[f(t, x(t))]^{\alpha}\right)=d\left(\left[f\left(t_{k}, x\left(t_{k}\right)\right)\right]^{\alpha},[f(t, x(t))]^{\alpha}\right)<\epsilon
$$

since $\left|t-t_{k}\right|<\lambda \leq \delta$,

$$
d\left([x(t)]^{\alpha},\left[x\left(t_{k}\right)\right]^{\alpha}\right) \leq d\left(\left[f\left(t_{k}, x\left(t_{k}\right)\right)\right]^{\alpha}, 0\right)\left|t-t_{k}\right|<M \lambda \leq \delta .
$$

Thus, by the definition of $D$, we have

$$
D\left(x^{\prime}(t), f(t, x(t))\right)<\epsilon
$$

on $\left|t-t_{0}\right|<\delta$ and $(t, x) \in J_{0}$.

Theorem 3.2 is completely proved.

ACKNOwLEDGement. This work has been supported by the Pusan National University, Korea, 1995.

\section{REFERENCES}

[1] R. J. Aumann, Integrals of set-valued functions, J. Math. Anal. Appl. 12 (1965), 1-12. MR 32\#2543. Zbl 163.06301. 
[2] C. Corduneanu, Principles of differential and integral equations, 2nd ed., Chelsea Publishing Co., Bronx, New York, 1977. MR 5512977.

[3] O. Kaleva, Fuzzy differential equations, Fuzzy Sets and Systems 24 (1987), no. 3, 301-317. MR 88j:34008. Zbl 646.34019.

[4] _ The Cauchy problem for fuzzy differential equations, Fuzzy Sets and Systems 35 (1990), no. 3, 389-396. MR 91f:34004. Zbl 696.34005.

[5] C. V. Negoita and D. A. Ralescu, Applications of fuzzy sets to systems analysis, John Wiley \& Sons, New York, Toronto, 1975, Translated from the Romanian. A Halsted Press book. MR 58 9442a. Zbl 326.94002.

[6] J. Y. Park and J. U. Jeong, Common fixed points of fuzzy mappings, Fuzzy Sets and Systems 59 (1993), no. 2, 231-235. CMP 94 06. Zbl 797.54022.

[7] J. Y. Park, Y. C. Kwun, and J. U. Jeong, Existence of solutions of fuzzy integral equations in Banach spaces, Fuzzy Sets and Systems 72 (1995), no. 3, 373-378. MR 96i:45006. Zbl 844.54010.

[8] M. L. Puri and D. A. Ralescu, Fuzzy random variables, J. Math. Anal. Appl. 114 (1986), no. 2, 409-422. MR 87f:03159. Zbl 592.60004.

Park AND Han: Department of Mathematics, Pusan National University, Pusan 609735, KOREA 


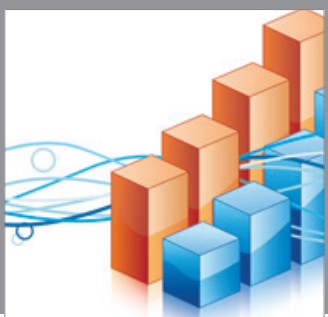

Advances in

Operations Research

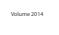

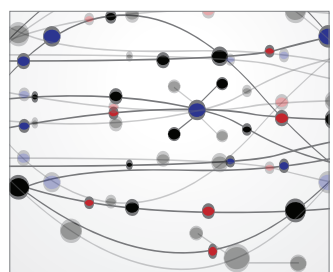

\section{The Scientific} World Journal
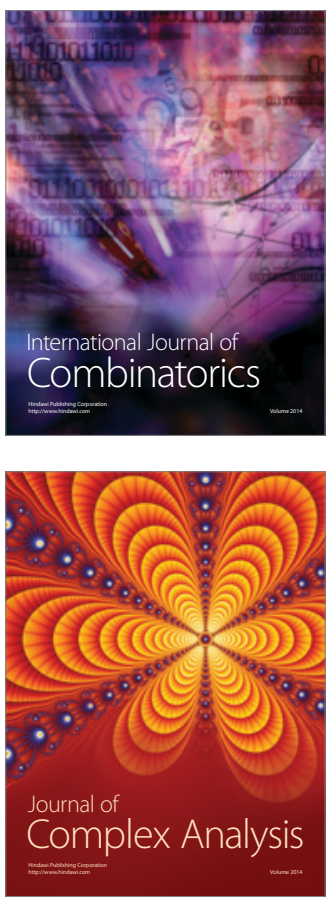

International Journal of

Mathematics and

Mathematical

Sciences
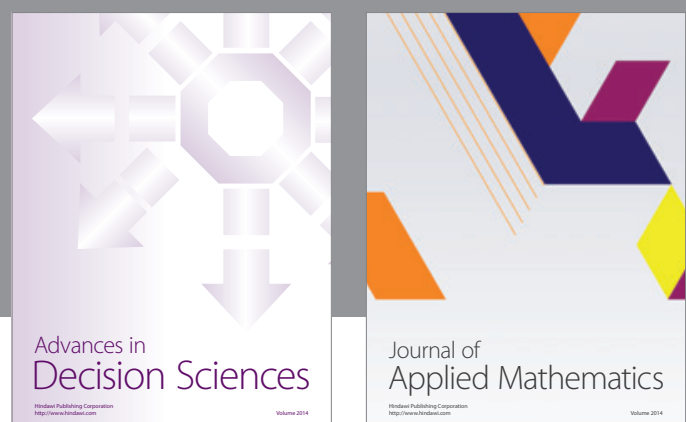

Journal of

Applied Mathematics
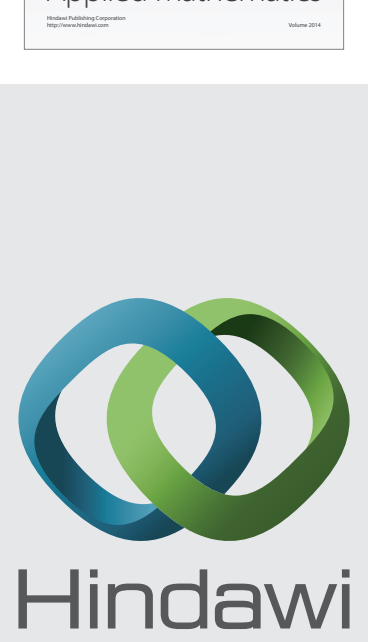

Submit your manuscripts at http://www.hindawi.com
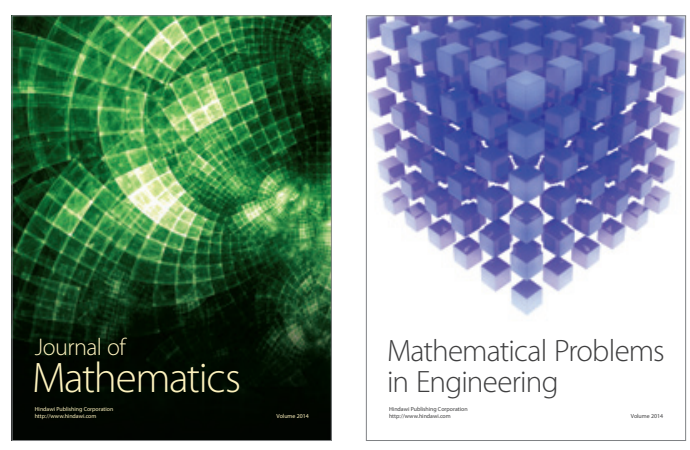

Mathematical Problems in Engineering
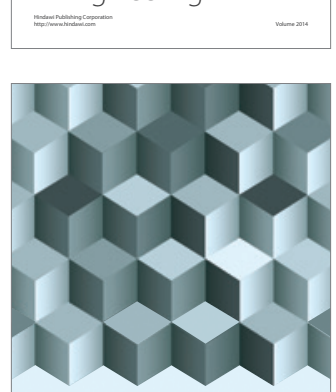

Journal of

Function Spaces
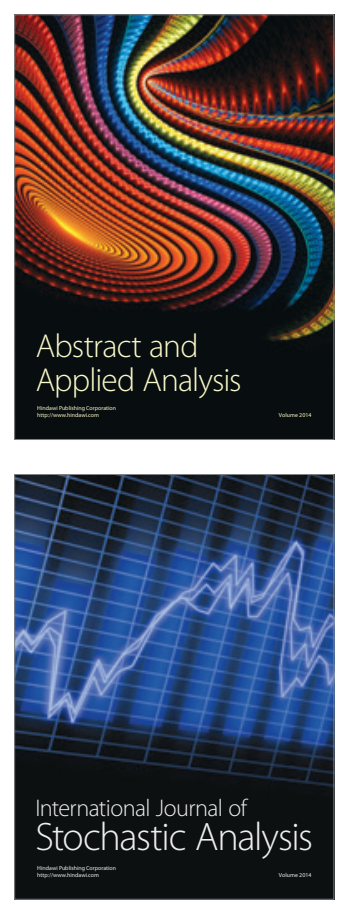

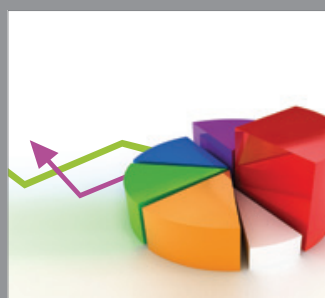

ournal of

Probability and Statistics

Promensencen
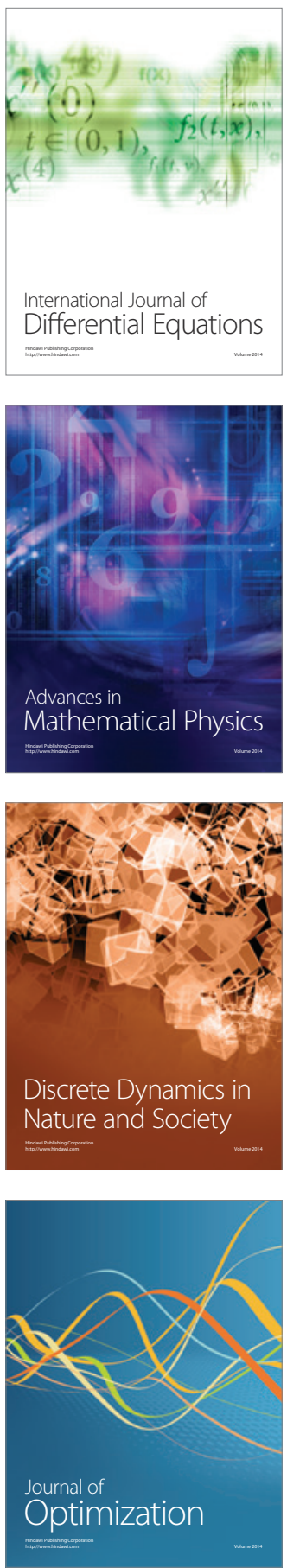